Pacific Journal of Mathematic 


\title{
A SIMPLE PROOF OF THE EXISTENCE OF MODULAR AUTOMORPHISMS IN APPROXIMATELY FINITE DIMENSIONAL VON NEUMANN ALGEBRAS
}

\author{
ROBERTO LONGO
}

\section{An elementary direct proof of Tomita-Takesaki Theorem for an AFD von Neumann Algebra.}

1. Introduction. After that $M$. Tomita [5] proposed the existence of the modular automorphisms several proofs of Tomita-Takesaki theorem have been given by Takesaki, van Daele, Haagerup (unpublished) and Zsido $[4,6,7,8]$, but none of these is elementary. However a simple proof of the theorem for approximately finite dimensional von Neumann algebras (with a cyclic'separating vector) may be extracted by an article of N.M. Hugenholtz and J.D. Wieringa 1], which was published very soon after the appearance of Tomita's original preprint. Motivated by the great interest that approximately finite dimensional von Neumann algebras have in Mathematics and in Physics, we present a simplified shorter version of the proof of Hugenholtz and Wieringa.

2. Statement and Proof. Let $\mathscr{R}$ be a von Neumann algebra acting on the Hilbert space $\mathscr{H}$ and $\xi \in \mathscr{H}$ a cyclic separating vector for $\mathscr{R}$ and then also for its commutant $\mathscr{R}^{\prime}$. As usual we introduce the antilinear operators

$$
\begin{aligned}
& S_{0}: A \xi, A \in \mathscr{R}, \rightarrow A^{*} \xi, \mathscr{D}\left(S_{0}\right)=\mathscr{R} \xi, \\
& F_{0}: B \xi, B \in \mathscr{R}^{\prime}, \rightarrow B^{*} \xi, \quad \mathscr{D}\left(F_{0}\right)=\mathscr{R}^{\prime} \xi ;
\end{aligned}
$$

$S_{0}$ (and $F_{0}$ ) is a closable operator: in fact if $A \in \mathscr{R}$ and $B \in \mathscr{R}^{\prime}$

$$
\begin{aligned}
\left(S_{0} A \xi, B \xi\right) & =\left(A^{*} \xi, B \xi\right)=(\xi, A B \xi)=(\xi, B A \xi) \\
& =\left(B^{*} \xi, A \xi\right)=\left(F_{0} B \xi, A \xi\right)
\end{aligned}
$$

so that $S_{0}^{*} \supset F_{0}$ and $\mathscr{D}\left(S_{0}^{*}\right)$ is dense.

In what follows we call $F=S_{0}^{*}$ the adjoint of $S_{0}, S=F^{*}$ the closure of $S_{0}$ and $\Delta=F S$ the modular operator which is non singular and positive. For the moment we suppose $\mathscr{R}$ finite dimensional; then there exists a faithful tracial state $\tau$ and for each state $\omega$ of $\mathscr{R}$ there exists a positive operator $H \in \mathscr{R}$ s.t. 


$$
\omega(A)=\tau(A H)=\tau\left(H^{1 / 2} A H^{1 / 2}\right), \quad A \in \mathscr{R},
$$

moreover $H$ is invertible iff $\omega$ is faithful. Let $\pi: \mathscr{R} \rightarrow \mathscr{B}\left(\mathscr{H}_{\tau}\right)$ be the GNS representation given by $\tau$ and $\omega$ a faithful state of $\mathscr{R}$ : we have $\mathscr{H}_{\tau}=\mathscr{R}, \pi(A) B=A B$ if $A, B \in \mathscr{R}$ and

$$
\omega(A)=\left(\pi(A) H^{1 / 2}, H^{1 / 2}\right), \quad A \in \mathscr{R},
$$

where $H^{1 / 2} \in \mathscr{R}$ is a cyclic separating vector for $\pi(\mathscr{R})$. It is easily seen the operator $\Delta$ of $\pi(\mathscr{R})$ relative to the vector $H^{1 / 2}$ is given by

$$
\Delta: A \in \mathscr{H}_{\tau} \rightarrow H A H^{-1} \in \mathscr{H}_{\tau}
$$

from which it follows

$$
\Delta^{-i t} \pi(A) \Delta^{i t}=\pi\left(H^{-i t} A H^{i t}\right), \quad A \in \mathscr{R}, t \in \mathbf{R},
$$

and then

$$
\Delta^{-i t} \pi(\mathscr{R}) \Delta^{t t}=\pi(\mathscr{R}), \quad t \in \mathbf{R} .
$$

By the uniqueness of the GNS representation we then see that for each finite dimensional von Neumann algebra $\mathscr{R}$ the modular operator $\Delta$ relative to a cyclic separating vector is such that

$$
\Delta^{-i t} \mathscr{R} \Delta^{i t}=\mathscr{R}, \quad t \in \mathbf{R},
$$

which is a particular case of Tomita-Takesaki theory.

Next step is proving the theorem when $\mathscr{R}$ is approximately finite dimensional, in the sense that there exists an increasing sequence $\mathcal{M}_{n} \subset \mathscr{R}$ of finite dimensional von Newmann algebras s.t.

$$
\mathscr{R}=\left(\bigcup_{n=1}^{\infty} M_{n}\right)^{\prime \prime}
$$

Then we have to prove:

THEOREM 1. Let $\mathscr{R}$ be an approximately finite dimensional von Neumann algebra acting on the Hilbert space $\mathscr{H}$ and $\xi \in \mathscr{H}$ a cyclic separating vector for $\mathscr{R}$. The modular operator $\Delta$ relative to $\xi$ is such that

$$
\Delta^{-i t} \mathscr{R} \Delta^{i t}=\mathscr{R}, \quad t \in \mathbf{R} .
$$

Our proof requires some lemmas. Let $\mathcal{M}_{n}$ be an increasing se- 
quence of finite dimensional von Neumann algebras generating $\mathscr{R}$ and put $\mathfrak{A}=\bigcup_{n=1}^{\infty} \mathcal{M}_{n}$ so that $\mathfrak{A}$ is a weakly dense $*$ subalgebra of $\mathscr{R}$.

Lemma 1. The linear subspace of $\mathscr{H} \mathfrak{A} \xi=\{A \xi \mid A \in \mathfrak{U}\}$ is a core for $S$.

Proof. It is enough to show that for each $A \in \mathscr{R}$ there exists a sequence $A_{n} \in \mathfrak{U}$ s.t.

$$
A_{n} \xi \rightarrow A \xi \quad \text { and } \quad A_{n}^{*} \xi \rightarrow A^{*} \xi
$$

and this follows because the selfadjoint elements of $\mathfrak{A}$ are dense in the selfadjoint elements of $\mathscr{R}$ in the strong topology.

Now we call $\mathscr{H}^{\prime}$ the domain of $S$ with scalar product

$$
(x, y)^{\prime}=(x, y)+(S y, S x), \quad x, y \in \mathscr{D}(S) .
$$

As the topology of $\mathscr{H}^{\prime}$ is that of the graph of $S$, we see that $\mathscr{H}^{\prime}$ is a Hilbert space and by lemma $1 \mathfrak{A} \xi$ is a dense linear subspace of $\mathscr{H}^{\prime}$. Now the sesquilinear form $(x, y), x, y \in \mathscr{H}^{\prime}$, is bounded in $\mathscr{H}^{\prime}$

$$
|(x, y)| \leqq\|x\|\|y\| \leqq\|x\|^{\prime}\|y\|^{\prime}, \quad x, y \in \mathscr{H}^{\prime}
$$

$\left(\|x\|^{\prime}=(x, x)^{1 / 2}\right)$ and therefore there exists a linear operator $T \in \mathscr{B}\left(\mathscr{H}^{\prime}\right)$ of norm less than 1 s.t.

$$
(T x, y)^{\prime}=(x, y), \quad x, y \in \mathscr{H}^{\prime}
$$

Let $E_{n} \in \mathcal{M}_{n}^{\prime}$ be the selfadjoint projection of $\mathscr{H}$ onto $\mathcal{M}_{n} \xi$ and $\mathcal{M}_{n E_{n}}=$ $\left\{\left.A\right|_{E_{n}(\mathscr{H})} \mid A \in \mathcal{M}_{n}\right\}$ the von Neumann algebra $\mathcal{M}_{n}$ cut down by $E_{n}$. The application

$$
\pi_{n}:\left.A \in \mathcal{M}_{n} \rightarrow A\right|_{M_{n \xi}} \in \mathcal{M}_{n_{E n}}
$$

is $a *$ isomorphism between $\mathcal{M}_{n}$ and $\mathcal{M}_{n_{E n}}$ because $\xi$ is a separating vector for $\mathcal{M}_{n}$; moreover $\xi$ is a cyclic separating vector for $\mathcal{M}_{n_{E_{n}}}$ and therefore if $S_{n}$ is the antilinear operator

$$
S_{n}: A \xi=\pi_{n}(A) \xi \rightarrow A^{*} \xi=\pi_{n}(A)^{*} \xi, \quad A \in M_{n}
$$

then, by what we know, the modular operator $\Delta_{n}=S_{n}^{*} S_{n}$ is s.t.

$$
\Delta_{n}^{-i t} \mathcal{M}_{n_{\mathrm{E} n}} \Delta_{n}^{i t}=\mathcal{M}_{n_{\mathrm{E} n}}, \quad t \in \mathbf{R} .
$$


We see also that $S_{n}=\left.S\right|_{\mu_{n \xi}}$ and if $\mathscr{H}_{n}^{\prime}$ is the linear space $\mathscr{D}\left(S_{n}\right)$ with scalar product

$$
(x, y)^{\prime}=(x, y)+\left(S_{n} y, S_{n} x\right), \quad x, y \in \mathscr{D}\left(S_{n}\right)
$$

then $\mathscr{H}_{n}^{\prime}$ is a Hilbert subspace of $\mathscr{H}^{\prime}$; as in (1) there exists $T_{n} \in \mathscr{B}\left(\mathscr{H}_{n}^{\prime}\right)$ of norm less that 1 s.t.

$$
\left(T_{n} x, y\right)^{\prime}=(x, y) \quad x, y \in \mathscr{H}_{n}^{\prime} .
$$

Lemma 2. Let $P_{n} \in \mathscr{B}\left(\mathscr{H}^{\prime}\right)$ be the selfadjoint projection of $\mathscr{H}^{\prime}$ onto $\mathscr{H}_{n}^{\prime}$. The operators $\tilde{T}_{n}=T_{n} P_{n}+\left(I-P_{n}\right) \in \mathscr{B}\left(\mathscr{H}^{\prime}\right)$ are s.t.

$$
\left\|T x-\tilde{T}_{n} x\right\|^{\prime} \rightarrow 0, \quad \forall . x \in \mathscr{H}^{\prime} .
$$

Proof. As $\bigcup_{n=1}^{\infty} \mathscr{H}_{n}^{\prime}=\mathfrak{A} \xi$ is dense in $\mathscr{H}^{\prime}$ by Lemma 1 , the orthogonal projections $P_{n}$ strongly converge to $I$ in $\mathscr{H}^{\prime}$ (we use the symbol $I$ to indicate both the identity of $\mathscr{H}^{\prime}$ and the identity of $\left.\mathscr{H}\right)$.

By (1) and (3)

$$
(T x, y)=\left(T_{n} x, y\right) \quad \text { if } \quad x, y \in \mathscr{H}_{n}^{\prime}
$$

and therefore

$$
\tilde{T}_{n}=P_{n} T P_{n}+\left(I-P_{n}\right)
$$

it follows that

$$
\left\|\tilde{T}_{n} x-T x\right\|^{\prime} \rightarrow 0
$$

if $x$ belongs to the dense subspace $\cup_{n=1}^{\infty} \mathscr{H}_{n}^{\prime}$ and then for each $x \in \mathscr{H}^{\prime}$ because the $\tilde{T}_{n}$ are equibounded. $\mathscr{H}$ by

We extend the modular operators $\Delta_{n}=S_{n}^{*} S_{n}$ to the whole space

$$
\tilde{\Delta}_{n}=\Delta_{n} E_{n}+I-E_{n}
$$

then each $\tilde{\Delta}_{n}$ is a positive invertible operator and we may consider $\tilde{\Delta}_{n}^{u}$, $t \in \mathbf{R}$.

LEMMA 3. For each real $t, \Delta^{t t}$ is the strong limit of $\tilde{\Delta}_{n}^{t}$ i.e.

$$
\left\|\tilde{\Delta}_{n}^{i t} x-\Delta^{i t} x\right\| \rightarrow 0, \quad x \in \mathscr{H} .
$$


Proof. The lemma is proved if we show that

$$
\left(\tilde{\Delta}_{n}+I\right)^{-1} \rightarrow(\Delta+I)^{-1} \quad \text { strongly; }
$$

in fact by a classical theorem on generalized convergence [3, Th. VIII. 20] it follows from (4) that

$$
f\left(\tilde{\Delta}_{n}\right) \rightarrow f(\Delta) \quad \text { strongly }
$$

for each bounded continuous complex valued function $f$ on the real line; moreover the same argument shows that (5) holds also when $f$ is bounded continuous on an open subset $A$ of the real line of spectral measure 1 for $\Delta$ and each $\tilde{\Delta}_{n}$; in particular for $A=(0, \infty)$ and $f(\lambda)=\lambda^{i t}$ the conclusion of the lemma follows from (4).

Note that the range of $(\Delta+I)^{-1}$ is equal to $\mathscr{D}(\Delta) \subset \mathscr{D}(S)$ so that we have by (1), for each $x, y \in \mathscr{D}(S)$,

$$
\begin{aligned}
\left((\Delta+I)^{-1} x, y\right)^{\prime} & =\left((\Delta+I)^{-1} x, y\right)+\left(S y, S(\Delta+I)^{-1} x\right) \\
& =\left((\Delta+I)^{-1} x, y\right)+\left(\Delta(\Delta+I)^{-1} x, y\right) \\
& =\left((\Delta+I)(\Delta+I)^{-1} x, y\right) \\
& =(x, y)=(T x, y)^{\prime}
\end{aligned}
$$

which implies

$$
T=\left.(\Delta+I)^{-1}\right|_{\mathscr{D}(S)}
$$

By the same argument $T_{n}=\left(\Delta_{n}+I\right)^{-1}$ and then

$$
\left.\tilde{T}_{n}\right|_{\mu_{n} \xi}=\left.\left(\tilde{\Delta}_{n}+I\right)^{-1}\right|_{\mu_{n} \xi}
$$

Applying Lemma 2, if $x \in \mathfrak{A} \xi$ we have for large $n$

$$
\begin{aligned}
\left\|\left(\tilde{\Delta}_{n}+I\right)^{-1} x-(\Delta+I)^{-1} x\right\| & \leqq\left\|\left(\tilde{\Delta}_{n}+I\right)^{-1} x-(\Delta+I)^{-1} x\right\|^{\prime} \\
& =\left\|\tilde{T}_{n} x-T x\right\|^{\prime} \rightarrow 0
\end{aligned}
$$

and as $\left\|\left(\tilde{\Delta}_{n}+I\right)^{-1}\right\| \leqq 1, n \in \mathbf{N}$, and $\mathfrak{U} \xi$ is dense in $\mathscr{H}$, we obtain the lemma.

By the isomorphism $\pi_{n}$ defined in (2) we may define the modular automorphisms $\sigma_{t}^{n}, t \in \mathbf{R}$, of $\mathcal{M}_{n}$ by

$$
\pi_{n}\left(\sigma_{t}^{n}(A)\right)=\Delta_{n}^{-i t} \pi_{n}(A) \Delta_{n}^{u}, \quad A \in \mathcal{M}_{n}, \quad t \in \mathbf{R}
$$


LEMmA 4. If $A \in \mathfrak{A}$ then the sequence $\sigma_{t}^{n}(A)$, defined above $a$ certain integer, strongly converges to $\Delta^{-i t} A \Delta^{i t}$, i.e.

$$
\left\|\sigma_{t}^{n}(A) x-\Delta^{-i t} A \Delta^{i t} x\right\| \rightarrow 0, \quad x \in \mathscr{H}, t \in \mathbf{R} .
$$

Proof. As we suppose $A \in \mathfrak{U}$ there exists $N \in \mathbf{N}$ s.t. $A \in \mathcal{M}_{n}$, $n \geqq N$. Take $x \in \mathfrak{A} \xi$ : there exists $N^{\prime} \in \mathbf{N}$ s.t. $x \in \mathcal{M}_{n} \xi, n \geqq N^{\prime}$. Then we have for $n \geqq \max \left(N, N^{\prime}\right)$

$$
\sigma_{t}^{n}(A) x=\pi_{n}\left(\sigma_{t}^{n}(A)\right) x=\Delta_{n}^{-i t} A \Delta_{n}^{i t} x=\tilde{\Delta}_{n}^{-i t} A \tilde{\Delta}_{n}^{i t} x
$$

and Lemma 3 implies

$$
\left\|\sigma_{t}^{n}(A) x-\Delta^{-i t} A \Delta^{i t} x\right\| \rightarrow 0, A \in \mathfrak{U}, x \in \mathfrak{U} \xi, t \in \mathbf{R} .
$$

As $\mathfrak{U} \xi$ is dense in $\mathscr{H}$ and $\left\|\sigma_{t}^{n}(A)\right\| \leqq\|A\|$ is an equibounded sequence the lemma follows.

Proof of Theorem 1. In view of Lemma 4 if $A \in \mathfrak{U}$ then $\Delta^{-i t} A \Delta^{i t}$, $t \in \mathbf{R}$, belongs to the strong closure of $\mathfrak{A}$ i.e.

$$
\Delta^{-u} \mathfrak{U} \Delta^{i t} \subset \mathscr{R}, \quad t \in \mathbf{R}
$$

by continuity

$$
\Delta^{-i t} \mathscr{R} \Delta^{i t} \subset \mathscr{R}, \quad t \in \mathbf{R}
$$

and then by symmetry

$$
\Delta^{-i t} \mathscr{R} \Delta^{i t}=\mathscr{R}, \quad t \in \mathbf{R} .
$$

REMARK 1. The essential tool we have used in the proof is the existence of a faithful tracial state on each approximating von Neumann algebra $M_{n}$

Acknowledgements. We are deeply indebted to S. Doplicher for encouragement and helping. We gratefully acknowledge the hospitality extended to us by Prof. L. Streit at ZiF, Bielefeld University in June-July 1976.

\section{REFERENCES}

1. N. M. Hugenholtz, and J. D. Wieringa, On Locally Normal States in Quantum Statistical Mechanics, Comm. Math. Phys., 11 (1969) 183-197. 
2. R. Longo, Dimostrazione del Teorema di Tomita-Takesaki sul Commutante e sugli Automorfismi Modulari di un'Algebra di von Neumann Iperfinita, Tesi dell Universita di Roma, (1975).

3. M. Reed, and B. Simon, Methods of Modern Mathematical Physics, I, Functional AnalysisAcademic Press, (1972).

4. M. Takesaki, Tomita's Theory of Modular Hilbert Algebras and its Application, Springer, (1970).

5. M. Tomita, Kyushu University preprint, (1967).

6. A. Van Daele, A Bounded Operator Approach to the Tomita-Takesaki Theory, Rome Meeting on $C^{*}$-Algebras and their Applications to Theoretical Physics, Symposia Mathematica, Academic Press, (1977).

7. _ـ Lectures given in Varenna summer school, (1973).

8. L. Zsido, A proof of Tomita's Fundamental Theorem in Theory of Standard von Neumann Algebras, Revue Comm. Pures et Appl., (to appear).

Received April 20, 1977. Supported in part by Consiglio Nazionale delle Ricerche (G.N.A.F.A.)

\author{
Istituto MATEMATICo \\ G. CASTLnUova \\ UNIVERSITÀ DI ROMA \\ 00100 ROMA
}





\section{PACIFIC JOURNAL OF MATHEMATICS EDITORS}

ICHARD ARENS (Managing Editor)

niversity of California

os Angeles, CA 90024

\section{. A. BEAUmont}

niversity of Washington

sattle, WA 98105

. C. MOORE

niversity of California

erkeley, CA 94720
J. DUGUNDJI

Department of Mathematics

University of Southern California

Los Angeles, CA 90007

R. FINN AND J. MiLgRAM

Stanford University

Stanford, CA 94305

\section{ASSOCIATE EDITORS}
F. BECKENBACH
B. H. NEUMANN
F. WOLF
K. YosHidA

\section{SUPPORTING INSTITUTIONS}

NIVERSITY OF BRITISH COLUMBIA

UNIVERSITY OF SOUTHERN CALIFORNIA

ALIFORNIA INSTITUTE OF TECHNOLOGY STANFORD UNIVERSITY

NIVERSITY OF CALIFORNIA

ONTANA STATE UNIVERSITY

UNIVERSITY OF HAWAII

NIVERSITY OF NEVADA

UNIVERSITY OF TOKYO

EW MEXICO STATE UNIVERSITY

UNIVERSITY OF UTAH

REGON STATE UNIVERSITY

NIVERSITY OF OREGON

WASHINGTON STATE UNIVERSITY

UNIVERSITY OF WASHINGTON

SAKA UNIVERSITY

AMERICAN MATHEMATICAL SOCIETY

The Supporting Institutions listed above contribute to the cost of publication of this Journal, but they a t owners or publishers and have no responsibility for its contents or policies.

Mathematical papers intended for publication in the Pacific Journal of Mathematics should be in typ Irm or offset-reproduced (not dittoed), double spaced with large margins. Underline Greek letters in re ierman in green, and script in blue. The first paragraph or two must be capable of being used separately as 'nopsis of the entire paper. Items of the bibliography should not be cited there unless absolutely necessary, hich case they must be identified by author and Journal, rather than by item number. Manuscripts, uplicate, may be sent to any one of the four editors. Please classify according to the scheme of Math. Revieu Idex to Vol. 39. All other communications should be addressed to the managing editor, or Elaine Bart Iniversity of California, Los Angeles, California, 90024.

100 reprints are provided free for each article, only if page charges have been substantially pai dditional copies may be obtained at cost in multiples of 50 .

The Pacific Journal of Mathematics is issued monthly as of January 1966. Regular subscription rate: \$72. year (6 Vols., 12 issues). Special rate: $\$ 36.00$ a year to individual members of supporting institutions.

Subscriptions, orders for numbers issued in the last three calendar years, and changes of address should ent to Pacific Journal of Mathematics, 103 Highland Boulevard, Berkeley, California, 94708.

UBLISHED BY PACIFIC JOURNAL OF MATHEMATICS, A NON-PROFIT CORPORATION

Printed at Jerusalem Academic Press, POB 2390, Jerusalem, Israel.

Copyright (C) 1978 Pacific Journal of Mathematics

All Rights Reserved 


\section{Pacific Journal of Mathematics}

\section{Vol. 75, No. $1 \quad$ September, 1978}

Mieczyslaw Altman, General solvability theorems

Denise Amar and Eric Amar, Sur les suites d'interpolation en plusieurs variables ..........................................

Herbert Stanley Bear, Jr. and Gerald Norman Hile, Algebras which satisfy a second order linear partial differential equation ..................

Marilyn Breen, Sets in $R^{d}$ having $(d-2)$-dimensional kernels ............

Gavin Brown and William Moran, Analytic discs in the maximal ideal space

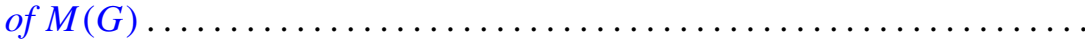

Ronald P. Brown, Quadratic forms with prescribed Stiefel-Whitney

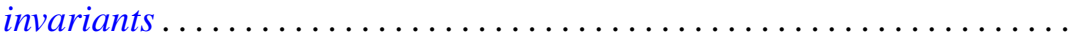

Gulbank D. Chakerian and H. Groemer, On coverings of Euclidean space by convex sets

S. Feigelstock and Z. Schlussel, Principal ideal and Noetherian groups.....

Ralph S. Freese and James Bryant Nation, Projective lattices ............

Harry Gingold, Uniqueness of linear boundary value problems for

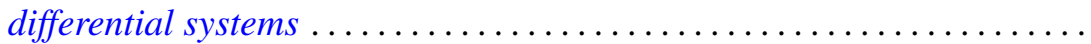

John R. Hedstrom and Evan Green Houston, Jr., Pseudo-valuation domains. . .

William Josephson, Coallocation between lattices with applications to measure extensions

M. Koskela, A characterization of non-negative matrix operators on $l^{p}$ to $l^{q}$ with $\infty>p \geq q>1$

Kurt Kreith and Charles Andrew Swanson, Conjugate points for nonlinear differential equations...........................

Shoji Kyuno, On prime gamma rings ........................ 185

Alois Andreas Lechicki, On bounded and subcontinuous multifunctions ..

Roberto Longo, A simple proof of the existence of modular automorphisms in approximately finite-dimensional von Neumann algebras ...

Kenneth Millett, Obstructions to pseudoisotopy implying isotopy for

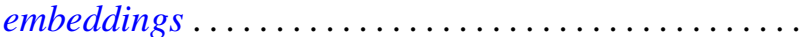

William F. Moss and John Piepenbrink, Positive solutions of elliptic equations. .

Mitsuru Nakai and Leo Sario, Duffin's function and Hadamard's

conjecture

Mohan S. Putcha, Word equations in some geometric semigroups ...

Walter Rudin, Peak-interpolation sets of class $C^{1} \ldots \ldots \ldots$

Elias Saab, On the Radon-Nikodým property in a class of locally convex

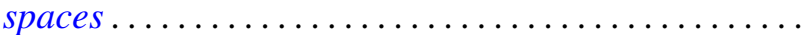

